\title{
An IT Service Management Literature Review: Challenges, Benefits, Opportunities and Implementation Practices
}

\author{
João Serrano ${ }^{1}$, João Faustino ${ }^{1, *}$, Daniel Adriano ${ }^{1}$, Rúben Pereira ${ }^{1}$ and Miguel Mira da Silva ${ }^{2} \mathbb{C}$ \\ 1 Department of Information Science and Technology, Instituto Universitário de Lisboa (ISCTE-IUL), \\ 1649-026 Lisboa, Portugal; jpsso11@iscte-iul.pt (J.S.); daniel_adriano@iscte-iul.pt (D.A.); \\ Ruben.Filipe.Pereira@iscte-iul.pt (R.P.) \\ 2 Instituto Superior Técnico, University of Lisbon, 1049-001 Lisboa, Portugal; mms@tecnico.ulisboa.pt \\ * Correspondence: jpcfo11@iscte-iul.pt
}

check for updates

Citation: Serrano, J.; Faustino, J.; Adriano, D.; Pereira, R.; da Silva, M.M An IT Service Management Literature Review: Challenges, Benefits, Opportunities and Implementation Practices. Information 2021, 12, 111. https://doi.org/10.3390/info12030111

Academic Editor: Willy Susilo

Received: 24 January 2021

Accepted: 1 March 2021

Published: 5 March 2021

Publisher's Note: MDPI stays neutral with regard to jurisdictional claims in published maps and institutional affiliations.

Copyright: (c) 2021 by the authors. Licensee MDPI, Basel, Switzerland. This article is an open access article distributed under the terms and conditions of the Creative Commons Attribution (CC BY) license (https:// creativecommons.org/licenses/by/ $4.0 /)$.

\begin{abstract}
Information technology (IT) service management is considered a collection of frameworks that support organizations managing services. The implementation of these kinds of frameworks is constantly increasing in the IT service provider domain. The main objective is to define and manage IT services through its life cycle. However, from observing the literature, scarcely any research exists describing the main concepts of ITSM. Many organizations still struggle in several contexts in this domain, mainly during implementation. This research aims to develop a reference study detailing the main concepts related with ITSM. Thus, a systematic literature review is performed. In total, 47 articles were selected from top journals and conferences. The benefits, challenges, opportunities, and practices for ITSM implementation were extracted, critically analysed, and then discussed.
\end{abstract}

Keywords: IT service management; systematic literature review; benefits; challenges; opportunities; implementation practices

\section{Introduction}

Nowadays, for most companies, information technology (IT) is one of the most important assets in the organizations' infrastructure. In fact, from early 1980s until now, the utilization and continuous improvement of IT has become an essential support to the business, regardless of the sector that organization belongs to [1]. This dependency on IT has been growing, as has its complexity, forcing organizations to increasingly have an effective management [2]. The management of IT operations has received more attention given its relevance on IT costs [3].

IT services have a big impact on the competitive advantage [4], being important to have an effective and efficient management. To do it, many IT organizations invest in IT service management (ITSM) frameworks such as the IT Infrastructure Library (ITIL) or the Control Objectives for Information and Related Technologies (COBIT) [5,6]. ITSM concentrates on IT operations, in particular in service delivery and support [7]. This "portfolio" of frameworks can produce several benefits to IT organizations, by helping them creating strategies and impelling fundamental changes, in order to become more adaptive, compliant and profitable [8].

If well implemented, ITSM frameworks can produce many benefits to organizations, such as decreases in operational costs and increases in operational efficiencies [5]. Some studies point that as process maturity levels grow, more benefits and lower issues organizations will face. These include a positive impact in business performance [9], an increase of organization profitability [10] and competitive leverage [11,12].

However, despite the benefits, many challenges have also been pointed out. Some claim that these frameworks are not easy to implement [13-15], criticizing the substantial complexity of the models and its abstraction in terms of knowledge and content $[13,16,17]$, not generating the benefits that are expected. Additionally, due to the fact that organizations 
need to implement different frameworks and standards to mitigate the difficulties and needs that only one standard or framework cannot address [18], the practices overlap each other $[19,20]$. Such overlap turns into an important issue since it increases organizations' costs, time, and resources [21,22]. These challenges generate opportunities for new research.

Based on previous paragraphs one may conclude that despite the proven benefits, challenges also exist regarding ITSM implementation, but new opportunities may arise with most recent versions of ITSM frameworks. Also, due to the evolution of new methodologies or cultures adopted by IT teams, ITSM frameworks need to be adapted to be able to work with these methodologies and cultures [23-25]. Thus, this suggests opportunities for improvement and adaptations to ITSM frameworks. So, based in previous statements the authors have reached to four concepts that are intrinsically connected in ITSM domain: challenges, benefits, opportunities, and implementation practices. It is possible to state from the existing literature that organizations may benefit from ITSM implementation; challenges can also be faced, which in turn can generate new opportunities.

Despite several investigations into the ITSM domain, and more organizations adopting it, the literature lacks a centralized consensus on benefits, challenges, and opportunities of ITSM implementation. This gap suggests an opportunity for a research to provide to practitioners the opportunity to know what to expect from an ITSM implementation, in terms of benefits, challenges and future opportunities. The present research adopted a systematic literature review (SLR) to review not only the ITSM concept but also to gather information about these four concepts in the ITSM area and their relations. This research intends to build solid foundations on these domains to assist further investigation in the ITSM domain. By doing so, this research can support organizations that intend to implement ITSM. This article centralizes all the findings of each concept, found in the SLR.

The present research is structured as follows. In Section 2, the contextualization of the IT Service Management concept is realized. Afterwards, in Section 3 the methodology adopted to perform the SLR is presented. Section 4, outlines all the filtration processes adopted by this research. In Section 5, some characteristics of the final article set are demonstrated. Then, in Section 6, all findings relating to each concept are presented. Subsequently, in Section 7, a discussion of each concept is realized. Finally, conclusions and future work are outlined in Section 8.

\section{IT Service Management}

The term service emerged in the 1930s when the U.S. Department of Commerce defined this concept to characterize economic sectors [7]. However, the notion of service and service management in the IT area just started to appear in the 1980s when the IT systems and all the IT environment increased in complexity [19]. IT services can be defined as a group of "tasks" [26] provided by an IT system or an IT department [27], that is, IT service can be characterized as the application of specialized capacities on IT assets [28]. IT services have a big impact on IT organization budget, being estimated that the maintenance and operability of these services are between $80-90$ percent of the total cost attributed to IT resources [4]. With the purpose of managing IT services, over years, IT organizations became interested in ITSM frameworks [29], since these frameworks are "specific servicesoriented best practices" [6].

The central focus of ITSM is the management of IT services [30]. In the earlier 1990s, with the increase in IT systems complexity, IT management became one of the main and more widely regarded functions in organizations. It was subsequently perceived that comprehensive management of IT was needed [31]. ITSM emerged from two main events: when the British Government developed the Information Technology Infrastructure Library (ITIL) framework, with the purpose of having better management and lower IT service costs [31], and with the creation of the service level management (SLM) framework [3]. Afterwards, with the demand and evolution of the IT domain, many other standards and frameworks were developed [32]. 
As mentioned before, the ITSM domain "has been developed to define, manage and deliver IT services" [33]. It is supposed that ITSM can be seen as a subset of Service Science [34] that concentrates on IT Operations [30]. ITSM can be defined as " ( . . ) an IT management framework that promotes service-oriented best practices to deliver value to organizations" [35], providing a methodical approach to the management of an IT service life cycle, from design, implementation, operation to continual improvement [30]. Not restricted to the operational costs, ITSM has also a great focus on the costs of the whole service life cycle [6].

ITSM models and standards have been created for the implementation and evaluation of processes [32], with ITIL being the most adopted framework by IT organizations $[17,27,34]$. Nevertheless, other frameworks and standards have been developed and applied such as the Microsoft Operation Framework (MOF), the Capability Maturity Model Integration for Services (CMMI-SVC) or even the ITSM main standard ISO/IEC 20,000 [36].

Several studies exist in literature approaching solutions for some of the ITSM challenges. As an example, Pereira et al. [37] have elaborated a maturity model for the Incident Management process by merging multiple frameworks and eliminating overlapped practices to mitigate said issues. Another example is the method created for implementing the ITIL framework, based on process management and simulation [38]. This method partially or completely supports organizations implementing the ITIL framework, serving as a solution for the implementation problem that organizations have.

\section{Research Method}

To enlighten the ITSM domain, this chapter details how the SLR methodology was performed. With all the selected articles, an overview of the ITSM area is presented. On the final section, an SLR summary is performed.

A literature review (LR) can be seen as an approach to evaluate and review the state of a certain domain [39]. The SLR is a methodology to conduct a rigorous and accurate $\mathrm{LR}$, providing a transparent and reproducible protocol so other authors can extend the review or to reach to the same or similar results [40,41]. This methodology is considered as a "systematic, explicit and reproducible method" with the purpose of "identifying, evaluating and synthesizing the existing body of completed and recorded work produced by researchers, scholars, and practitioners" [42]. By adhering to this kind of methodology, the LR quality will be improved in several ways [43].

With the intention of reviewing the ITSM main concepts and seeking its benefits, challenges and opportunities, an SLR was conducted. This review will help new researchers to have an easily accessible unification of ITSM information and might be a starting point for investigators to develop new solutions for opportunities or challenges found. This research is in accordance with the guidelines for conducting an SLR created by Kitchenham [44], as can be seen in Figure 1 . 


\begin{tabular}{|c|c|c|}
\hline $\begin{array}{c}\text { Outlining Systematic } \\
\text { Literature Review }\end{array}$ & $\begin{array}{l}\text { Conductimg Systematic } \\
\text { Literature Review }\end{array}$ & Reporting the Review \\
\hline $\begin{array}{l}\text { Identification of the need for } \\
\text { a review } \\
\text { Despite ITSM importance the } \\
\text { literature lacks a study capable } \\
\text { of synthesize the pros and cons } \\
\text { of its implementation as well } \\
\text { as future directions } \\
\text { Objective of the review } \\
\text { Gather information about the } \\
\text { main benefits, implementation } \\
\text { practices, challenges and } \\
\text { opportunities of ITSM } \\
\text { Review protocol } \\
\text { Search string, filters, } \\
\text { repositories and inclusion } \\
\text { criteria }\end{array}$ & $\begin{array}{l}\text { Applying filters and get final } \\
\text { articles } \\
47 \text { articles. } \\
\text { Perform Data extraction } \\
\text { - Sample characteristics; } \\
\text { - Information extraction from } \\
\text { selected articles. }\end{array}$ & $\begin{array}{l}\text { Summarize the data extracted } \\
\text { Summarizing the information } \\
\text { obtained. } \\
\text { Reporting the findings } \\
\text { Conclusions of ITSM domain }\end{array}$ \\
\hline
\end{tabular}

Figure 1. Systematic Literature Review Steps.

\section{Outlining Systematic Literature Review}

This SLR has not only the objective to perform an ITSM overview but in addition to detail all the challenges, benefits, opportunities, and implementation practices that companies are currently facing. More precisely, this research intends to answer the following research questions:

- RQ.1: What are the main ITSM benefits?

- RQ.2: What challenges organizations have been struggling with during the implementation of ITSM?

- RQ.3: What research opportunities exist in the ITSM field?

- RQ.4: What are the best implementation practices to implement the ITSM framework?

To proceed with the SLR and collect proper information to pursue the established objective, answering the formulated research questions, five electronic repositories were selected:

- IEEE Online Library (https://ieeexplore.ieee.org/Xplore/home.jsp (accessed on 4 March 2021));

- Google Scholar (https:/ / scholar.google.com/ (accessed on 4 March 2021));

- $\quad$ SpringerLink (https://link.springer.com (accessed on 4 March 2021));

- Elsevier (https://www.sciencedirect.com (accessed on 4 March 2021));

- $\mathrm{ACM}$ (https://dl.acm.org (accessed on 4 March 2021)).

Next, specific keywords in conjunction with the AND and OR operators were adopted. The search string is presented below.

"IT Service Management" AND

("Frameworks" OR "Best Practices" OR "Standards" OR "Main Concepts" OR "Difficulties" OR "Benefits" OR "Advantages" OR "Positive effects" OR "Barriers" OR "Disadvantages")

The review included only English and Portuguese articles and accepted articles exclusively published on Journals, Scientific Magazines, and Conferences Proceedings. Lastly, no date filter was used. 
In the first phase, with the selected keywords in each repository, without any filter, a search was carried out. After that, four filters were created. Since each of the electronic libraries uses different "search approaches", a keyword adaptation for each repository was used. It should be mentioned that in Google Scholar, considering that it does not have a filtering option, and that it is not possible to download all the results at once, each article was added to the Atlas support tool manually.

The description of the filters applied in this SLR is as follows: the 1st filter applies the keywords on the article title, on the abstract or on the author keywords; The 2nd one has the objective of removing duplicate articles; with the 3rd filter, articles that are published in lower ranked publications/journals are removed. For that reason, two websites, Scimago (Scimago website: https://www.scimagojr.com/ (accesse on 4 March 2021)) and Conference Ranks (Conference Ranks website: http:/ / www.conferenceranks.com/ (accesse on 4 March 2021)), were used. Each respectively provide journal and conference ranks. For conferences, only A, B, A1, A2, B1, and B2 ranks of ERA and Qualis rankings were accepted. When an article was assessed by both rankings, the Qualis ranking prevailed. For journals only Q1 and Q2 ranks were accepted. Finally, the last stage of filtration was done by assessing article introduction and conclusion. Since this last filter had a "subjective judgement", the following criteria inclusions were followed:

- Only articles about the ITSM theme were accepted.

- Only articles about benefits, good practices, disadvantages/problems and opportunities of the ITSM area were accepted.

\section{Conducting a Systematic Literature Review}

As aforementioned, the selected articles needed to progress through four filters in each repository. The filtration process, by each online repository and each filter, is visible in Table 1.

Table 1. Results of the Filtration Process.

\begin{tabular}{cccccc}
\hline & No Filter & 1st Filter & 2nd Filter & 3rd Filter & 4th Filter \\
\hline IEEE & 1104 & 647 & 614 & 132 & 6 \\
ACM & 110 & 35 & 20 & 5 & 3 \\
SpringerLink & 1337 & 45 & 45 & 28 & 5 \\
ScienceDirect & 6903 & 189 & 189 & 143 & 11 \\
Google Scholar & 200 & 200 & 129 & 64 & 22 \\
Total & 9654 & 1116 & 997 & 372 & 47 \\
\hline
\end{tabular}

Furthermore, the articles from the Google Scholar repository were added manually. Taking into account that this repository cannot extract the final set of articles at once, the 1 st filter was not applied in this repository.

The 1st filter had the intention of splitting the articles that are exclusively related with the ITSM domain from those who just referred to these concepts in the article body. This was completed by just selecting the ones that had the keywords in the title, abstract and author keywords. These three article sections were chosen for being the main parts that summarize the article's subject matter. With this filter, it was possible to reject a considerable number of articles. The 2 nd filter had the goal of eliminating duplicate articles. On the 3rd filter, 957 articles were ranked by their publication rank, which totaled to nearly 389 publications given that various articles had the same publication (conference/journal). Consequently, in the 4 th filter, 372 articles were read and assessed with the inclusion criteria described above, which resulted in 47 final articles. The flow of the filtration process can be seen in Figure 2. 


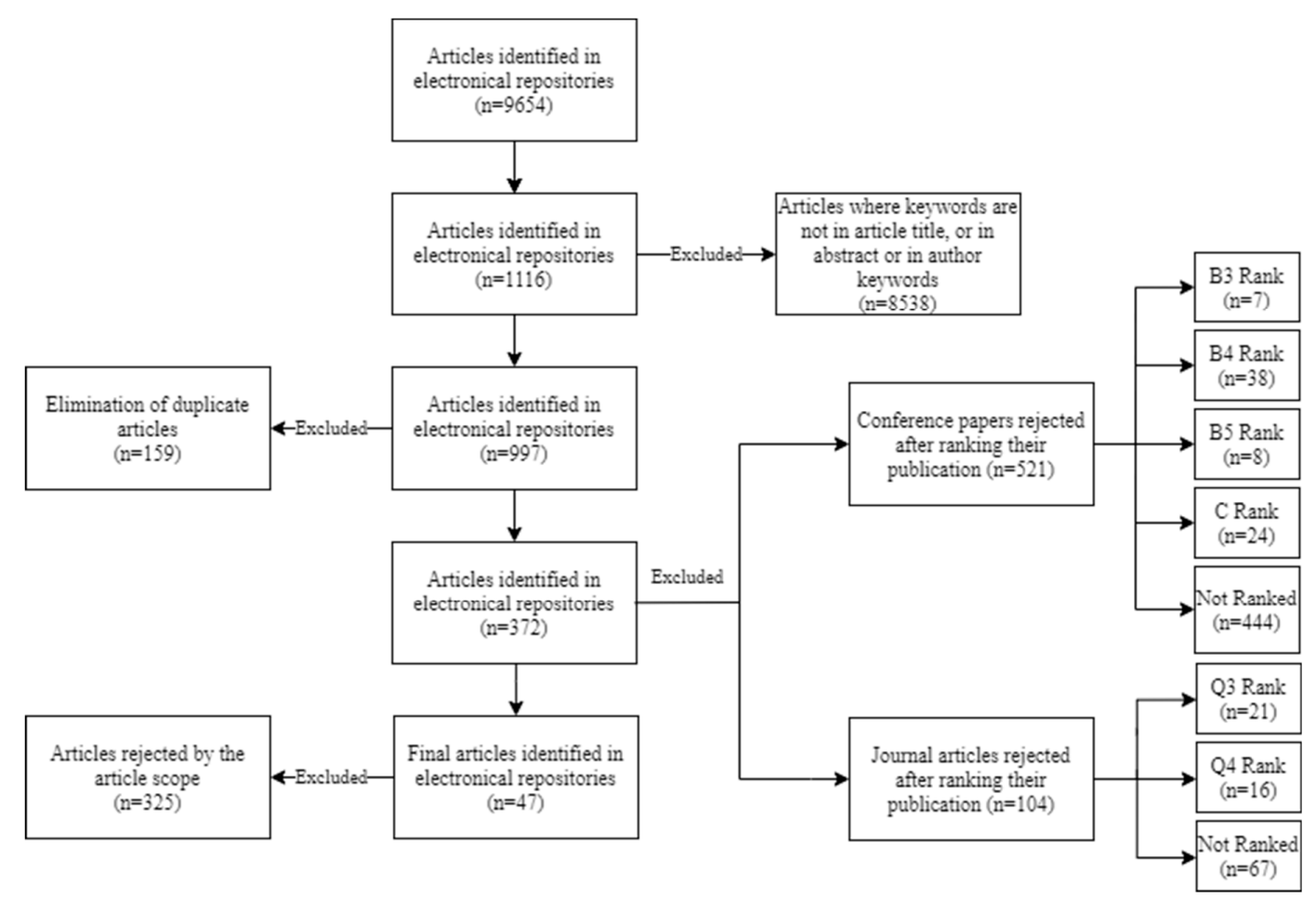

Figure 2. Flow of the Filtration Process.

\subsection{Sample Characteristics}

The final set of articles was composed of 47 publications. As mentioned before, only articles from conference proceedings and journals were accepted. As can be observed in Figure 3, which represents the distribution of the articles by their publication rank, only the $B$ rank of ERA ranking is absent of publications amongst the final set.

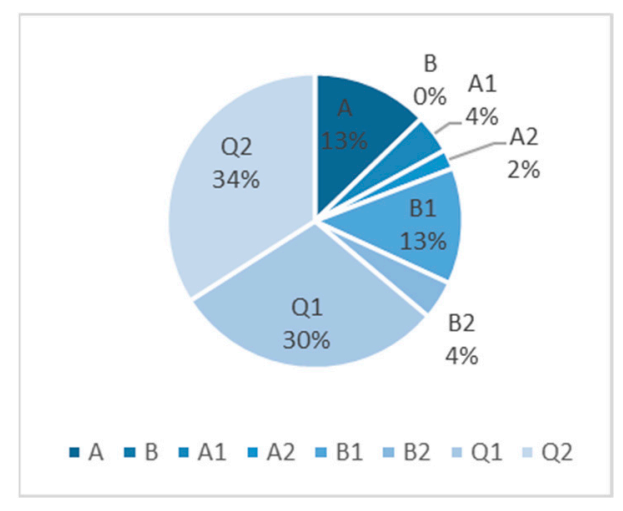

Figure 3. Articles Percentage by Publication Rank.

The distribution of the total number of each publication type is visible in Figure 4. The journal articles were the main contributor, being nearly $62 \%$ of the final set of articles. The journal or conference that stands out is the Journal of Computer Standards \& Interfaces, with five publications present on the final article set.

Figure 5 lists the field's evolution in the scientific community since 2010 (https:// scholar.google.com/ (accessed on 4 March 2021)), demonstrating the relevance of the domain while Figure 6 shows the distribution of the articles by year. It can be observed that the year interval goes from 2002 to 2019. Considering the evolution of the articles throughout the years and the article's sample in study, it can be stated that over the last 
three years, articles related to the four chosen ITSM concepts have decreased, emphasizing the need for a centralized article with all the ITSM concepts reunited.

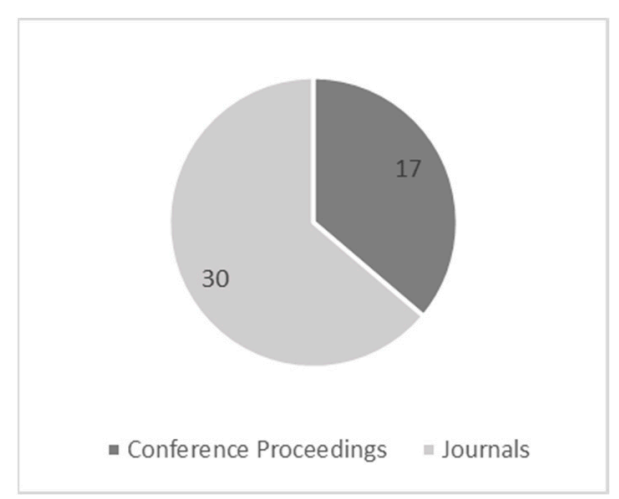

Figure 4. Articles Percentage by Publication Type.

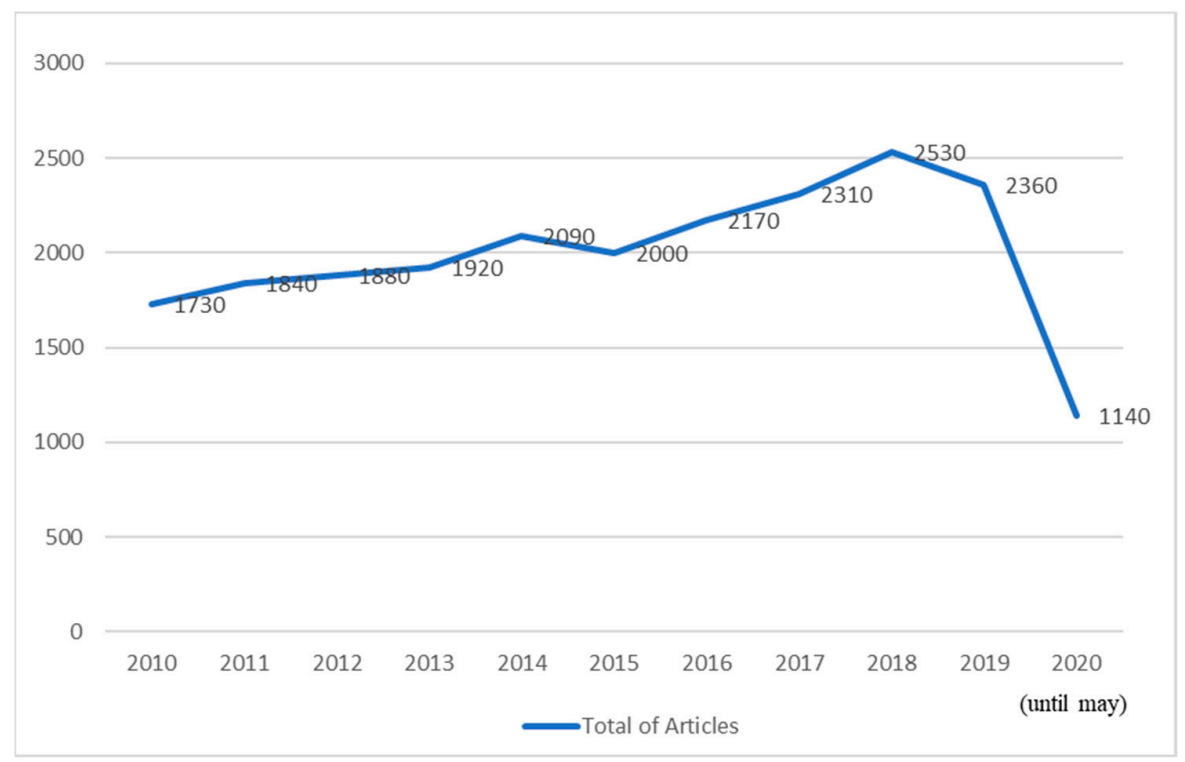

Figure 5. Total of ITSM Articles Published Since 2010.

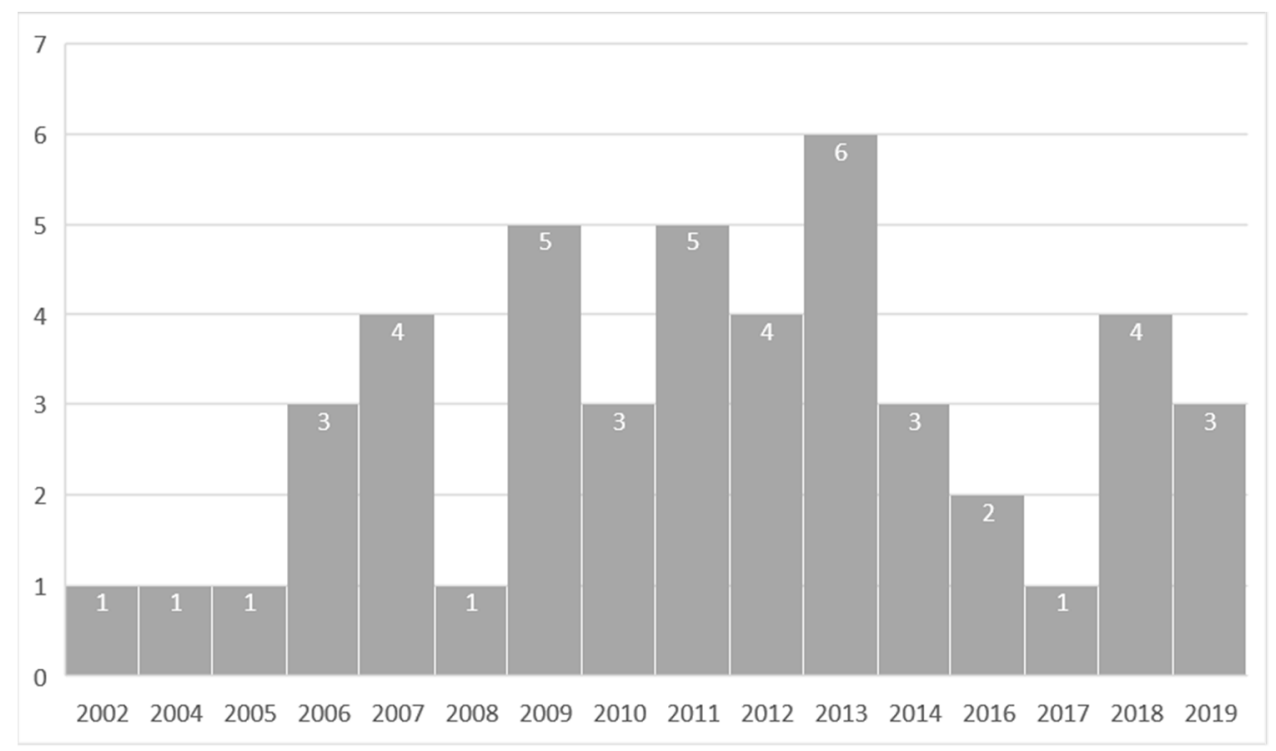

Figure 6. Articles by Year (final set). 


\subsection{Information Extraction Process}

After applying the filters, the final set of articles were read. To support the information extraction from these articles, software for qualitative analysis was used. As mentioned before, all the details surrounding the ITSM domain were extracted.

\section{Reporting the Review}

\subsection{ITSM Benefits}

When implemented in a planned and "conscious" way, ITSM frameworks can produce many organizational benefits. As mentioned before, an SLR methodology was followed. After the analysis of the selected researches, the authors have summarized and characterized the benefits (Table 2).

ITSM frameworks support organizations in having a proper management strategy [45]. In fact, throughout the articles, it is commonly described that these frameworks help Management in developing better processes documentation [5] and monitoring [11], allowing for more detailed audits and IT process reports [33]. Moreover, the enhancement of documentation and monitoring brings higher transparency and comparability for the organization [11], producing an increase of processes maturity [38] and allowing management to have greater control of the processes, supporting organizations situated in environments of uncertainty [6].

Table 2. ITSM Benefits.

\begin{tabular}{cc}
\hline Benefits & Articles \\
\hline Better processes control/documentation & {$[2,5,6,11,33,34,38,46-48]$} \\
Tangible improvements in process metrics (i.e., incident resolution & {$[1,2,6,11,33,45,46,48,49]$} \\
times, change implementation time, predictable failures) & {$[5-7,11,29,33,38,45,49]$} \\
IT service quality improvement & {$[5,6,11,33,34,38,49,50]$} \\
Increase of customer satisfaction & {$[2,5,7,19,29,33,38,49]$} \\
Decrease in IT expenses & {$[1,2,5,11,28,38,49,51]$} \\
Higher efficiency/performance & {$[6,7,11,28,38,46,48]$} \\
Better IS-business alignment & {$[5,28,29,46-48]$} \\
sharing efficiency & {$[6,7,19,20,33,52]$} \\
Efficiency in the internal communication process/information & {$[1,38,46,51]$} \\
Increase of organizational competitiveness & {$[5-7,33]$} \\
Mature processes & {$[5,33]$} \\
Increase of organization revenue & {$[5]$} \\
Better employee satisfaction & Reduction in staff \\
\hline
\end{tabular}

Most IT organizations implement ITSM frameworks due to the most perceivable improvements that these kinds of "tools" produce in the organization [2]. Many of these observable improvements occur at an operational level [6], where processes metric improvements captured by the organization are easily perceptible [11]. Also, it is defended that this type of benefit improved infrastructure predictability, reducing server or application faults [46]. Additionally, some research refers that incidents and error volume is drastically decreased [33] given that, with processes implemented, problems that may arise during normal operations can be dealt with proactively [45].

Furthermore, some authors also argue that the quality of IT services provided is increased [38] by a continuous-improvement method [7], increasing IT service flexibility and adaptability [29].

The increase of service quality mentioned by the scientific community is a generic benefit since the quality is increased due to the better control and performance of the processes. However, these quality increments generate one of the most important benefits to an organization: customer satisfaction [11]. It was described that customer satisfaction is enhanced as the quality of framework's activities is improved [38], satisfying the present 
and future demands of customers [33]. Additionally, these frameworks support customers with higher availability, responsiveness, and expertise [5].

In addition, the proactivity of dealing with adverse and unfavorable events that may appear might prevent wastes of money [2], being this benefit one of the most important for higher management. The articles affirm that applying ITSM principles can lead to a substantial decrease in operation and maintenance costs $[7,19]$, such as in service provision costs [38], being intrinsically connected with the increment of the infrastructure and operational efficiency [5].

In fact, ITSM practices come under investigation for organizations that desire to increase the effectiveness of their functions [28]. It is argued that, with ITSM, organizations processes become more mature, thus turning the performance of operational activities more robust [1]. Also, adjusting organizational practices to the implemented processes can help organizations increase productivity [51].

Moreover, another major benefit found was the alignment that the ITSM applies between the IS and the vision and strategy of the organization [28]. ITSM frameworks not only provide benefits at an operational level but also help organizations situate themselves in a more strategic position [6]. Such alignment further contributes to improving the effectiveness of the company [38].

By adopting ITSM frameworks and having the processes well-established and defined, communication is enhanced through all organizational layers, providing a "common language" [28]. This type of approach improves the consultation between groups within an organization [5] and increases the effectiveness and service quality of IT providers [29].

It is important for companies not to be mere support for the business but to also demonstrate attentiveness to new opportunities and needs of the "global" market [6]. With the implementation of ITSM frameworks and standards, organizations recognize opportunities to improve organizational competitiveness [7] in terms of client confidence and/or reputation [33] or even in relation to international competition advantage [20].

Another benefit pointed in literature is that ITSM frameworks turn the organizations' processes more mature [46] and consequently improve productivity [51]. By having internal guidelines provided by these frameworks, it is possible to develop and automate standard processes [11], helping to establish and enable higher maturity towards a better vision of the processes and their global understanding [38].

Most of the companies implement ITSM frameworks not just help them in having better service management but to increase revenue as well [5]. This relates to previous benefits since the reduction of operational costs and the increase of service quality translates into new customers, which subsequently converts into the increment of organizational revenue. Posteriorly, it is defended that the companies can easily return the investment made [6] and have greater financial control [33].

Some authors also point out that ITSM frameworks help in the transparency and robustness of companies' internal procedures [5]. By having improved and well-established "protocols", employee satisfaction will increase [33], which will support achieving expectations of IT staff [5]. Having employees pleased and confident not only helps organizations reorient or change their culture, but also improves company productivity.

Last but not least, perhaps the most controversial benefit present in literature is the reduction of staff. Although being found only in one article, it is stated that, given the frameworks' ability to increase process maturity, via partial or total automation, staff may be relocated or even reduced [5]. In fact, several attempts have been carried out to automate ITSM processes [53], having contemplated the concern of relocating staff [54].

\subsection{ITSM Challenges}

Despite the benefits that ITSM can produce, many organizations still face a variety of challenges daily. The challenges found in the literature (Table 3) were gathered and analysed. 
Table 3. ITSM Challenges.

\begin{tabular}{cc}
\hline Challenges & Articles \\
\hline Organization resistance & {$[2,29,30,47,48,50,55,56]$} \\
Frameworks complexity & {$[1,15,19,20,36,47]$} \\
Lack of knowledge/skills & {$[1,15,19,29,30,55]$} \\
Processes assessment (costly and time-consuming) & {$[4,5,20,35,57,58]$} \\
Lack of management support & {$[29,30,48,50,59]$} \\
Lack of resources & {$[29,30,56,59]$} \\
Difficult on quantifying the benefits & {$[5,29,48,56]$} \\
Big investment needed (Implementation and maintaining momentum) & {$[3,5,11,30]$} \\
Hard of planning to implement multiple frameworks & {$[20,21,33,52]$} \\
Steady lower costs & {$[49]$} \\
\hline
\end{tabular}

As mentioned by Keel et al. [2], the major challenges in ITSM can be divided into four areas: technology, data, process and people. In fact, resistance to change from co-workers is the most cited challenge. It is defended that this resistance is due to organizational culture [55], associated with the difficulties in changing and implementing a new strategy at the organizational level [48]. This general and natural tendency of people to resist change triggers a substantial "mentality" challenge for management [56]. It can also cause delays in implementation [48]. To sum up, such problems are due to organizational culture that lacks subjacent values like innovation or even support for change. To adopt a substantial change, management should prepare the technical staff first.

Additionally, ITSM frameworks are criticized by their complexity. Some of the critics point to the broad and generic definitions that the frameworks have [14,19]. Plus, the frameworks and standards do not provide guidelines for implementing them [36]. Furthermore, although the frameworks characterize the flows and interactions between processes, they lack a solution for process measurement and improvement [20].

Analysing Table 3, one can see that most of the challenges refer to the framework's implementation phase. Despite the growth interest in ITSM frameworks, they still lack clear implementation guidelines, creating many challenges for IT organizations.

Moreover, the lack of knowledge/skills of ITSM frameworks is another big challenge that organizations are facing [15]. In the literature, it is pointed out that "a gap between the knowledge of ITSM frameworks and their implementation" exists [1], which demonstrates that this challenge is intrinsically connected with framework complexity. In addition to that, the inexperience of project managers or core members of the organization [29] turn the implementation and maintenance of the practices difficult to execute [55].

Many IT organizations that have an ITSM framework implemented consider process assessments a difficult challenge, with this "act" of assessment important for continuous ITSM improvement [57]. However, process assessment methods are expensive and timeconsuming [35]. Additionally, the frameworks do not have a measurement system for process improvement that could help organizations [20]. In the authors view, an organization without an assessment tool can lead to failures and will not be beneficial in mid-term, since an improvement strategy will not be defined because the as-is state of the processes is unknown.

Another challenge cited in the literature was the lack of management support. It is referenced in a case study that, although the "management layer" provided a big endorsement to the implementation, the commitment was not adequate [59]. In this case, the implementation failed. Also, it is defended that the lack of management support can create delays in the implementation process [48], which can increase the associated costs. Furthermore, without the support of senior management, the implementation could not be in compliance with the organizations' vision and strategy [50].

In light of this research, lack of management support is inherent to the lack of resources challenge. The big difficulty in framework implementation is the definition of the right "amount" of resources to provide [29]. Without management support, gathering sufficient 
resources, not just for the implementation, but also for process maintenance, can become a significant challenge [56].

Justifying the cost of the implementation and the investment of a framework by trying to show the benefits can be considered as a challenge [56]. Despite potential benefits, it is defended by literature that the implementation of frameworks can be slowed, not only by the amount of investment, but also due to the difficulty of realising the benefits and linkage between operational "performance measurement" and the organizations' revenue [5]. Quantifying the benefits can turn into a difficult task, and can be a reason for lack of continual investment [48].

It is estimated that IT service costs are between $70 \%$ and $80 \%$ of IT organization expenditure [30], therefore a big investment is needed, this being an enormous organizational challenge. The implementation of a framework will require needed investment given that the entire organizational "way of working" will change [3]. In addition to that, an extra cost and investment should be considered given that by running necessary infrastructure, the continual improvement and continuous performance monitoring also has associated costs [11].

Several organizations, with the purpose of being compliant with regulations and for process improvement, have the requirement of implementing several frameworks/standards [20]. In fact, this is becoming a challenge for organizations, given that planning these implementations requires taking into account the integration and interoperability of both frameworks for cost savings, complexity reduction and efficiency [52]. Also, the process' practices of various frameworks overlap each other $[19,33,37]$, increasing the need for a good strategy when implementing multiple frameworks.

Finally, improving and maintaining the quality of provided services is an important task, not just for revenue but for continual ITSM improvement. However, it has been stated that it is challenging to maintain constant or lower costs while improving delivery quality [49].

\subsection{ITSM Opportunities}

As mentioned before, organizations face challenges when attempt to implement ITSM which increases their difficulty along the process. As Albert Einstein once sad "in the middle of every difficulty lies an opportunity". In this sense, this section does not have the objective to introduce solutions for problems that organizations are confronting, but to list some opportunities worthy of further research (Table 4).

Table 4. ITSM Opportunities.

\begin{tabular}{cc} 
Opportunities & Articles \\
\hline Lack of guidelines to processes improvement & {$[15,60-62]$} \\
Processes assessment & {$[4,58,60]$} \\
Identifying processes interdependencies and their overlap & {$[20]$} \\
Maturity Models for the needs of IT management providers & {$[17]$} \\
Cloud computing and DevOps & {$[49]$} \\
Lack of guidelines to processes improvement & {$[15,60-62]$} \\
\hline
\end{tabular}

Processes improvement is important for organizations [60]. However, organizations still struggle with improvement and in defining what to improve [14]. It is defended that process capability grows and costs are reduced with increasing maturity levels [61], that is, improved processes can reduce costs to organizations. A research with the purpose of creating guidelines to improve processes could save resources to IT organizations.

As stated above, organizations find it difficult to assess their processes; ITSM frameworks do not provide guidelines to help on this matter. Most assessments are conducted by third-party organizations and have a big associated cost [4]. Also, it is criticized that the guidelines that do exist lack transparency [58]. It is visible that a gap exists between the implementation of processes and their assessment. 
The "phenomenon" of adopting multiple frameworks can create opportunities. Some experienced organizations are doing business with this "phenomenon", by providing services that help organizations with multiple frameworks, reducing their complexity. A potential opportunity lies in understanding the process interdependencies and linkage, to help determine the overlap between each process [20]. This research can help organizations that need to implement various frameworks and processes, to have a better plan to understand which processes overlap and their relative dependencies.

Many maturity models have been developed. These kinds of tools can be useful for organizations that want to assess a certain domain in their organization. Machado et al. [17] argued that maturity models still lack in meeting the needs of IT management providers and appoints that a maturity model that would have a focus on service improvement could help organizations enhance their processes and services. Grounded on the previous statements, further investigation relating ITSM and the "maturity model" concept should be incentivized.

Although cloud computing is not a new concept, it is starting to be used and related to several domains. In fact, cloud computing allows for higher performance. IT service implementation could be faster via the utilization of cloud computing and DevOps [49]. Solutions with the intercalation of both areas could not just decrease implementation times, but also provide computing infrastructure to small organizations that would help in maintaining service operability.

\subsection{ITSM Implementation}

The implementation process of an ITSM framework is one of the most significant challenges faced by organizations, as observed in Section 6.2. This research has collected some advisable practices found in literature (Table 5) to help ITSM implementation.

Table 5. ITSM Implementation Practices.

\begin{tabular}{cc}
\hline Implementation Practices & Articles \\
\hline Commitment from all levels of management & {$[2,11,48,59]$} \\
IT and business processes must be aligned & {$[1,28,47]$} \\
Follow-up and assessment & {$[4,11,47]$} \\
Quick wins & {$[11,48]$} \\
Training & {$[1,11]$} \\
Commitment from all levels of management & {$[2,11,48,59]$} \\
\hline
\end{tabular}

When an organization moves to adopt a new ITSM framework, it is important to have the commitment from all levels of management [2]. Senior management support is considered a critical success factor in any major IT project [59]. In fact, research has mentioned that in five case studies, all guaranteed that for successful implementation senior management should support the project, which would help to fund and provide the right resources for implementation [48].

Also, planning the implementation is an essential phase. One of the most important practices found when implementing a framework is the consideration of the IT strategy and vision of the organization when planning the adoption of a framework [28]. Additionally, the implementation must be aligned with business objectives, in order not only to solve a problem or need, but to also create a positive impact in the strategy of the organization [1]

Creating and having an improvement and follow-up strategy established is also mentioned as a good practice [47]. Having follow-up and implementation plans will help in the successful and effective implementation at the operational level [4]. Defining process improvement strategies can help organizations to have mature processes and achieve cost savings.

When planning an implementation, seeking quick-wins can be a good option [11]. It is defended that there is no correct order to implement processes, although looking for 
quick wins can be a good strategy [48], which will help the organization achieve visible benefits at a faster rate.

Before performing a big change in the way that an organization works, staff must be advised and trained. Training is considered one of the most important tasks before the implementation of a framework [1]. The affected staff must be prepared, and a personnel development plan must be enforced in order to qualify co-workers in terms of mentality and knowledge [11].

\section{Key Findings}

\subsection{RQ.1: ITSM Benefits}

The present research has analysed studies developed by the scientific community and collected 13 benefits. By observing

Table 2, it is visible that all these benefits are connected, where several benefits translate into others. Many of the identified benefits are tangible in nature, being the easiest to perceive. Most of the organizations implement ITSM frameworks to help them better manage their infrastructure and services. It is important to note that these tools create an "environment" of certainty where all processes are well-defined and co-workers know what it is necessary to perform to accomplish specific goals, that is, inside the organization what must be done is known. This can turn the IT infrastructure of an organization more robust and increase employee satisfaction, therefore improving company performance.

With this "atmosphere" of efficiency, where the bulk of conditions to have a strong infrastructure with controlled processes are reunited, in a natural way, the quality of provided services by these companies will grow. This can be considered the central benefit of ITSM framework application. Some companies also aim to increase their revenue and new customers, however, to achieve this it is necessary to have more flexible and robust services.

Moreover, service quality is dependent on its "surroundings", that is, to reach a high level of service quality it is necessary to create an effective infrastructure, including welldocumented and settled processes and also an IT staff that is motivated and trained to perform their tasks.

As mentioned before, the raising of service quality will create satisfied customers as well as bring new ones, consequently increasing revenue while decreasing operational costs. When correctly implemented, there is prevention of unnecessary waste of money by employees, and by possible errors and problems that may appear. Conceptual models can be useful to demonstrate the flows of information and to give a high level "picture" of themes [63] in a way that can easily demonstrate the correlation and complexity between concepts [64]. From this perspective and by observing the connection and correlation between the benefits, the authors decided to interpret these connections and a conceptual model for the ITSM benefits was developed (Figure 7).

Since the benefits produce other benefits and are connected, the expression leads to was used. Following the line of reasoning described before, by having an environment where the processes are controlled and well-defined, not just communication efficiency increases but also employee satisfaction is improved upon, since knowledge of what to do is more widely known.

Also, possessing controlled processes coupled with their well-definition, will originate mature processes [11]. Tangible improvements in process metrics can be achieved by mature processes [17,45], creating here an important "conceptual triangle" with these three benefits. Considering that the processes are at a certain level of maturity, metrics can be used to adopt continual improvement, enabled greater process control. This "conceptual triangle" can be seen as an improvement cycle.

Several investigations detail that as process maturity increases, business performance will also be improved $[6,9,10]$. Following the conclusions of these articles, the authors defined that mature processes originate higher efficiency and performance in organiza- 
tions [5,17]. Additionally, with mature processes, the waste of resources will decrease,

being correlated with the benefit of "decrease in IT expenses" [5,7,61].

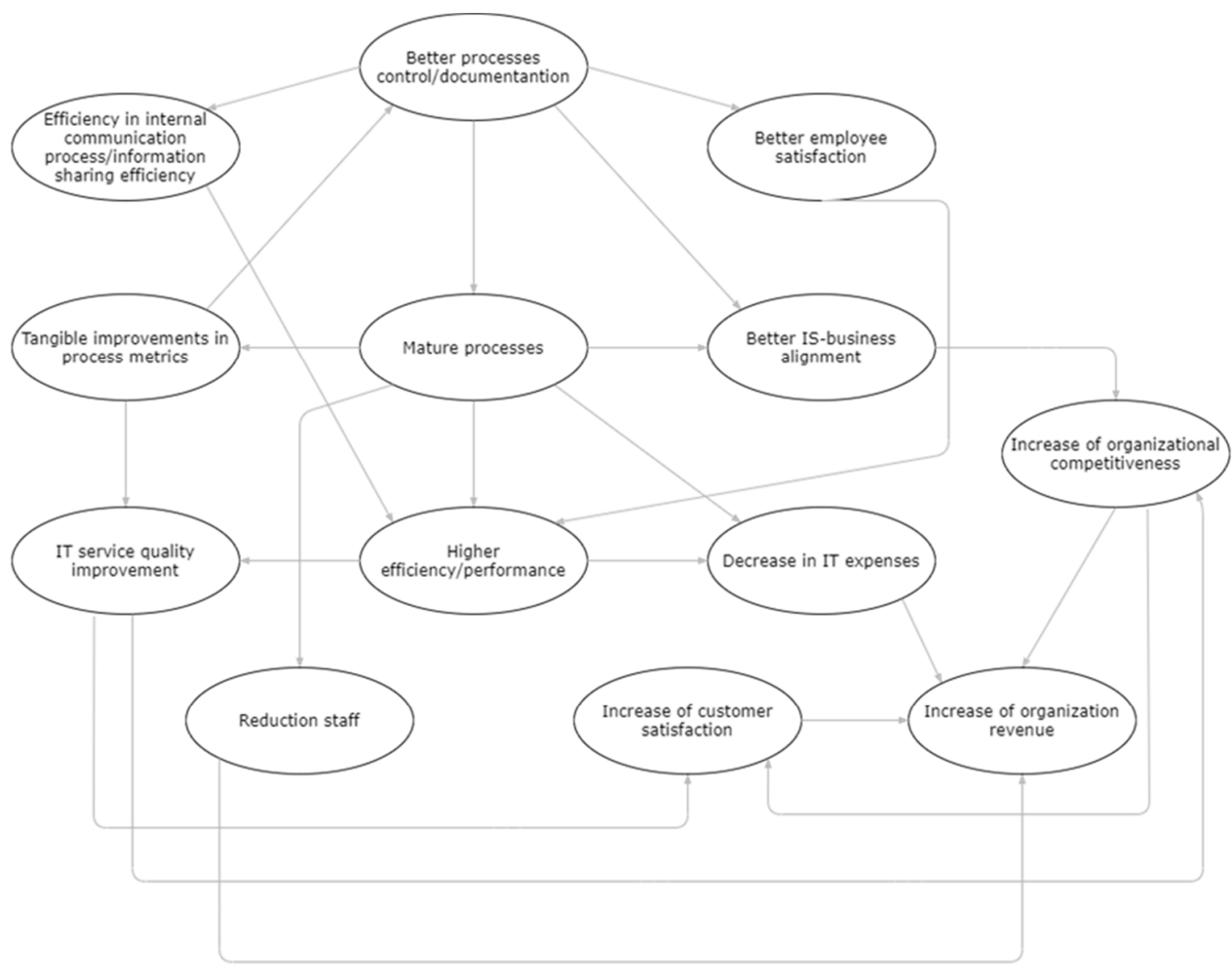

Leads to

Figure 7. ITSM Benefits Conceptual Model.

With all the metrics of processes controlled and with higher performance, the quality of services provided by the organization will grow as a result [11,38]. As mentioned before, this benefit is considered by the authors of this research the central one, since this is one of the main objectives of ITSM frameworks, and the principal benefit to increase the customer satisfaction and bring more clients to the organization $[33,38]$.

By having customers pleased and via the achievement of gaining new clients, revenue generated in the organizations will rise. Besides that, the fact of having IT expenses decreased coupled with potential staff reduction, which occurs as a result of process maturity and automation, this will also contribute to the increment in revenue of organizations. Frequently, this benefit is the principal objective for higher management to implement an ITSM framework.

Additionally, having controlled processes supports the IS-business alignment [6], helping organizations to have strategic objectives aligned with IT infrastructure, which will create and grow competitiveness [6] in the organization since a robust and efficient infrastructure, prepared for any change in the vision, strategy or prepared for any demand, is in place [19]. This condition can help organizations achieve higher competitiveness in an international environment.

It is perceivable that having controlled processes can originate an interconnected "chain of benefits". It is important to note that, by observing the developed conceptual model, the main identified benefits are correlated with others, however, those benefits are 
originated by others, that is, a benefit will not produce other benefits without first being improved itself.

With this in mind, and with the fact that several benefits are dependent on the "maturity" of other benefits to achieve a good mature level, the authors developed a table (Table 6) that presents which benefits promote (out) and are promoted by (in) other benefits.

Table 6. Relation between Benefits and Inputs/Outputs.

\begin{tabular}{ccc}
\hline Benefits & In & $\rightarrow$ Out \\
Mature processes & 1 & 5 \\
Better processes control/documentation & 1 & 4 \\
Tangible improvements in process metrics (i.e., incident resolution times, & 1 & 2 \\
change implementation time, predictable failures) & 2 & 2 \\
IT service quality improvement & 3 & 2 \\
Higher efficiency/performance & 2 & 2 \\
Increase of organizational competitiveness & 2 & 1 \\
Increase of customer satisfaction & 2 & 1 \\
Decrease in IT expenses & 2 & 1 \\
Better IS-business alignment & 1 & 1 \\
Efficiency in the internal communication process/information sharing & 1 & 1 \\
Better employee satisfaction & 1 & 1 \\
Reduction in staff & 4 & 0
\end{tabular}

Given the identified relationship amongst benefits, it is reasonable to argue that organizations should first focus on the benefits that promote (out) more other benefits rather than being promoted (in) by other benefits. Therefore, by observing Table 6, the benefit "Mature processes" can be considered one of the most important to achieve, since on its own it can trigger five further benefits.

On the other hand, the last benefit organizations should be focused on and aiming to improve is the "Increase of organizational revenue", given that it does not promote other benefits, and is promoted by or generate from other four benefits.

Although being the last benefit to aim, as we mentioned earlier, it is also the goal that most organizations desire. This may somehow justify why a considerable part of ITSM implementation fail [25,50,59]. Organizations may have not been focused on the right priorities to achieve the final aim. This highlights the fact that to achieve substantial benefits, all the environment around ITSM must be well understood and implemented holistically, being imperative not to focus merely on revenue growth (main goal).

Table 6 can be compared to a "Quick-Wins" strategy, where the objective is to demonstrate which benefit should be addressed first in order to achieve further benefits.

\subsection{RQ.2: ITSM Challenges}

Despite benefits, a not planned and haphazard adoption of ITSM frameworks can create many challenges and problems. Nevertheless, by observing the challenges in Table 3, it is possible to affirm that most challenges faced by organizations are before and during implementation. It is noted that people face a big challenge in the implementation and operability of an ITSM framework. The application of frameworks changes the way how collaborators work, creating a change-resistant environment. In fact, this challenge transcends to any type of organization in which employees are comfortable with the manner that they work, feeling uncomfortable to change. Most of this resistance to change can be considered a result of organizational culture that underlines the organization itself, lacking the "right principles" to this kind of action.

The lack of management support is pointed out as a nuclear challenge for ITSM framework implementation. It stands out that most of the challenges are due to the fact of lacking management support, which without said support a lack of resources or even 
a lack of an "example" to operational co-workers is generated, producing in that way a possible resistance to change. In addition, the lack of support from management can be directly connected with the big investment needed to implement a framework, and with not having enough budget to invest.

Also, one of the main challenges is the actual complexity of frameworks. Nowadays, frameworks do not describe an implementation process, making it challenging for organizations that do not have adequate knowledge or skills to adopt them. Companies, in an attempt to apply a framework with lacking knowledge, can have disastrous results in revenue. Furthermore, these frameworks do not characterize how processes should be assessed or even improved upon, not increasing the processes maturity and not producing the benefits that are expected, probably creating the challenge of being difficult to quantifying the benefits.

When taking everything into account there still exists lack of information in frameworks targeting the implementation, assessment and improvement of processes, generating a loss of money for organizations, consequently creating doubt by the management level of a company.

In this section, a conceptual map was also created (Figure 8) to illustrate the relationships and associations among the challenges found in literature. As aforementioned, challenges, if not mitigated, can lead to other challenges, creating a difficult environment for a successful ITSM implementation and operation.

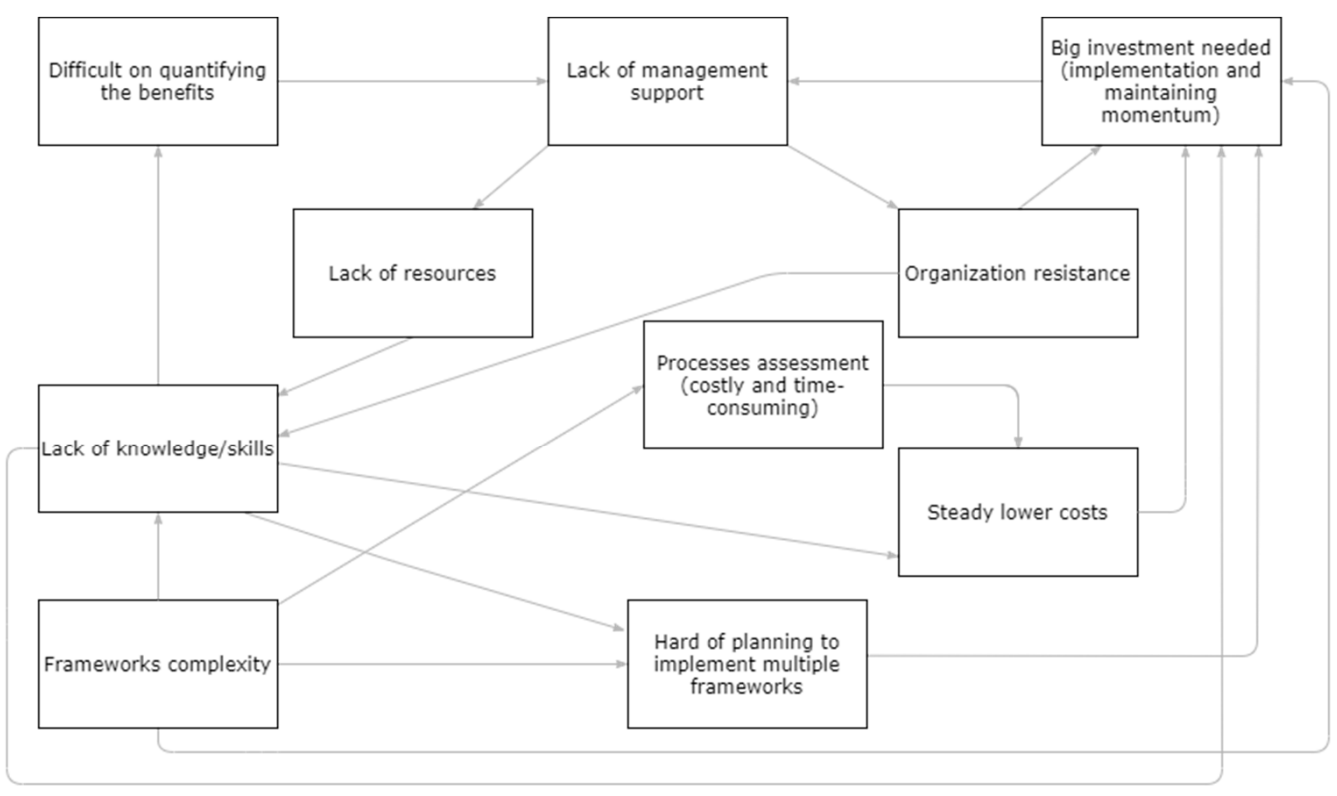

Figure 8. ITSM Challenges Conceptual Model.

Lack of management support in organizations leads to a lack of resources $[29,50]$, since it is the management that decides where to apply all the means that companies have. Besides that, as mentioned, this lack of support can promote an environment of resistance from operational lines [48], since the organizations' managers do not take the lead in giving an "example".

Considering that organizations have no sufficient resources applied in ITSM, it will create a lack of knowledge and skills, given the fact that there is no funds to invest in training that would help to understand and acquire the "know-how" of ITSM. However, not only the lack of resources leads to this challenge, but also organizational resistance, since the co-workers would not intend to apply themselves in this kind of training.

Having the knowledge and the skills of ITSM can help collaborators justify to management the use and implementation of ITSM, by demonstrating the benefits that it can produce. However, without awareness and understanding, it can become difficult to 
quantifying the benefits, leading to the challenge of lack of management support [50]. Additionally, with lack of ITSM know-how, it can be hard to steadily lower costs of operational lines, creating in this situation a waste of resources. Also, for organizations that need to implement multiple frameworks, this can turn into a big issue when they do not have the appropriate knowledge and skills for that type of implementation.

Besides the fact that most of the challenges found are based on the bad practices that an organization "adopts", the ITSM framework complexity is considered an external challenge, given that it is not produced by organizations. Organizations and ITSM experts have been criticizing the ITSM frameworks for being too generic and complex. This challenge leads to the hard planning of an implementation and also results in lack of knowledge. Furthermore, these frameworks are criticized for not supporting and demonstrating how organizations should assess their processes [47,57], being difficult to improve and produce maturity [20], which by itself will not create the benefits that should be created.

Also, the frameworks' complexity can lead to a need of a big investment, not just for the ITSM implementation but also for its maintenance, considering that for organizations that do not have the "know-how" mistakes can easily be made. The implementation of ITSM needs a big investment of organizational resources since companies will change the way they operate. However, with lack of good practices, a waste of resources will happen, and further big investments will be needed to overcome committed errors. This challenge, in the authors view, is one of the reasons for lack of management support in the provision of proper resources to implement and maintain ITSM.

As done with the benefits, a table with the relation between the challenges was developed (Table 7). Contrary to the benefits, observing Table 7 we will note that the challenges that have more arrows coming out are the ones that should be avoided since these challenges will produce many more.

Table 7. Relation between Challenges and Inputs/Outputs.

\begin{tabular}{ccc}
\hline Challenges & $\rightarrow$ In & $\rightarrow$ Out \\
\hline Frameworks complexity & 0 & 4 \\
Lack of knowledge/skills & 3 & 4 \\
Organization resistance & 1 & 2 \\
Lack of management support & 2 & 2 \\
Processes assessment (costly and time-consuming) & 1 & 1 \\
Lack of resources & 1 & 1 \\
Difficult on quantifying the benefits & 2 & 1 \\
Hard of planning to implement multiple frameworks & 2 & 1 \\
Steady lower costs & 5 & 1 \\
\hline
\end{tabular}

\subsection{RQ.3: ITSM Opportunities}

Additionally, this article has gathered five opportunities from the research community. Despite being few in numbers, the opportunities focus mostly on the problems that organizations face with ITSM. Observing Table 4, the opportunities for research are related mainly with the assessment of the processes. Since organizations should have a continual improvement plan to produce benefits that should be created with the implementation of ITSM, for the authors this opportunity is considered as of great importance.

Both opportunities of processes assessment and improvement are intrinsically connected with the opportunity of developing maturity models for the ITSM domain. Maturity models support organizations to assess their processes and practices, by assigning a level of maturity to a process that is being evaluated in accordance with the practices established by the model. In fact, several maturity models have been developed for ITSM $[37,64,65]$. However, other possibilities could be studied, since many organizations still have difficulties in the assessments, and maturity models have been criticized.

Nevertheless, cloud computing was mentioned as an opportunity for the future of ITSM, by helping small or medium organizations with the resources that they could 
originally not afford. With the ITSM opportunities, considering that those found were small, the authors could not produce a conceptual model with the connections and correlations between them.

\subsection{RQ.4: ITSM Implementation}

Finally, since organizations face challenges during ITSM implementation, five practices were found. Looking at Table 5 it is possible to conclude that management support in ITSM implementation is one of the most crucial practices, considering that it is one of the main factors that can dictate if the implementation will have the proper resources.

Additionally, using quick wins with a strategy to choose which processes should be implemented, supports organizations on having the infrastructure aligned with the business and prepared for the demand.

Also, the training and preparing of co-workers for the change is important as well. From this research's' view, training practice to staff could mitigate the challenge of resistance to change in organizations.

As occurred with the opportunities, it was not possible to correlate the best implementation practices; consequently, a conceptual model for the implementation practices was not developed.

As mentioned before, the complexity of the frameworks can be considered as an external challenge, being difficult to prevent. Another challenge that should be avoided is the lack of knowledge/skills, given that this challenge "defines" how well ITSM will be implemented and operated.

Also, it is important to mention that the challenge of "Big investment needed" is the challenge that is more dependent on others. A big investment to change organizations will always be needed; however, in the view of the authors, to decrease the value of this investment, all other challenges surrounding it must be mitigated or controlled.

This table can help an understanding of which challenges should be mitigated first to produce the fewest challenges possible.

\subsection{ITSM Environment and Contextualization}

The best implementation practices can mitigate several challenges and produce benefits. Also, as mentioned before, challenges can create opportunities for research. A connection exists between all these concepts. Having this in mind, the authors created a conceptual model with the relationships among all concepts (Figure 9).

The best practice of training is connected to both challenges and benefits. Training not only helps organizations achieve the knowledge of how to implement ITSM but also how to maintain it. By training collaborators, they will gain the proper "know-how" of how to operate the processes [1,29], increasing their maturity and improving their metrics, making ITSM processes more efficient. Additionally, training co-workers can help partially mitigate the external challenge of framework complexity. Also, with collaborators knowing what-todo, the waste of resources will decrease; consequently, the investment in implementation and operability will also decrease.

As mentioned earlier, the "Quick-Wins" strategy is considered a good practice of implementation, for those who do not know which processes to implement. From the research's' point of view, this strategy is able to exhibit benefits, mitigating the difficulty of showing ITSM benefits [11], since the seeking for earlier benefits underlines this strategy. Furthermore, this can decrease the investment needed to implement ITSM. A Quick-Wins strategy also produces several benefits, mainly the enhancement to IS-business alignment given that a choice is made to implement the processes that "fits" better in the organization's IT infrastructures. Also, this strategy may increase the organization's revenue by creating several benefits in an earlier stage of ITSM implementation and operation. 


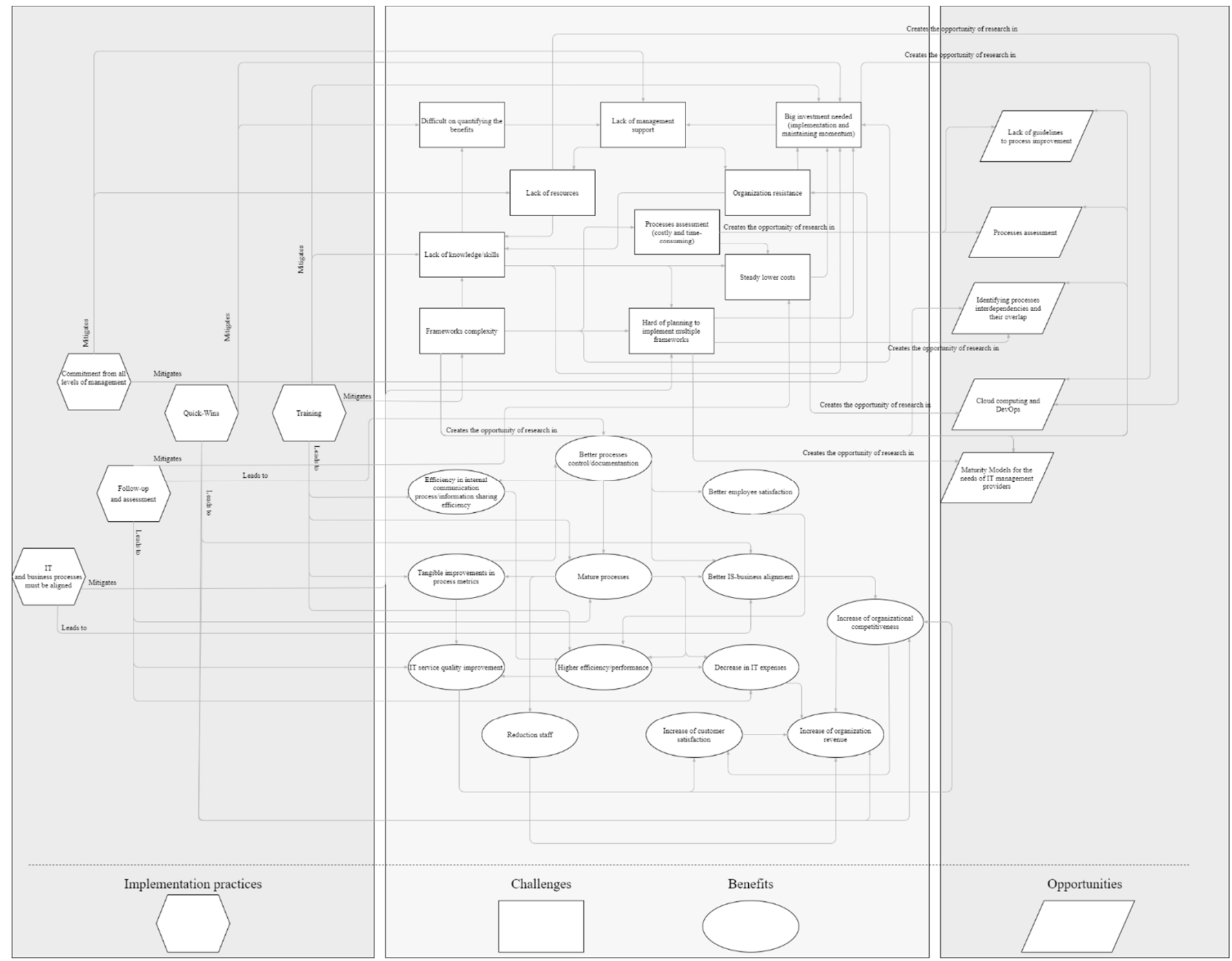

Figure 9. Conceptual Model of ITSM Main Concepts.

The commitment of all management hierarchy is not necessarily connected with the benefits found in the literature. However, it is intrinsically correlated with the mitigation of several challenges, which by itself produces benefits. As demonstrated before, with all levels of management committed with this big project, the resistance of the organization to change could be avoided $[2,29,48]$, and the lack of resources that should be provided to the ITSM implementation and operation could be mitigated [59].

The follow-up and assessment of the processes is viewed as a practice with a perspective towards continual improvement [60]. This practice not only helps organizations have processes well-documented but well controlled as well. The assessment will allow for the development of process maturity [29], since organizations will have a basis that will dictate where processes must be improved [4]. Additionally, the follow up should support organizations on the process's operability, helping to decrease the waste of resources, mitigating the challenge of maintaining steady lower costs and producing the benefit of decreasing IT expenses.

Many organizations have the necessity of implementing more than one framework face to the regulations of the market [20]. However, this kind of implementation brings many difficulties since it is necessary to have a good plan defined. Having the processes aligned with the business and with the organizations' strategy, can help organizations partially mitigate hard planning and implementation. Also, this good practice produces the benefit of having IS aligned with the organization's business. 
As aforementioned, challenges originate opportunities for research. Although several practices could mitigate various challenges, there still exists the necessity of new research for the creation of innovative and new solutions. The integration of DevOps and Cloud computing is a good example for research. Several organizations still have a lack of resources for the implementation of ITSM [49], and cloud providers, with the provision of proper resources, could help small and medium organizations have an infrastructure that would make possible to have ITSM allocated. Also, the difficulty of making costs stable and low should be an opportunity for investigation in conjunction with cloud computing.

Companies still have the difficulty of assessing their own processes due to framework complexity. Also, with the requirement of implementing several frameworks, some organizations face a variety of challenges [20]. The authors found that the junction between maturity models and ITSM could help organizations mitigate these kinds of challenges [17]. Furthermore, these opportunities are also related to the lack of guidelines for assessment and improvement that frameworks do not provide.

This conceptual model demonstrates how the four concepts relate to each other. Moreover, this map could help organizations on planning their implementation and have a plan of mitigation for the future challenges that they could face.

\section{Conclusions}

Traditional IT organizations have evolved. This evolution made organizations change from technology providers to service providers, looking at IT management with a different perspective. ITSM is a conjunction of frameworks that help IT service providers enhance the management practices of their services. Additionally, ITSM supports organizations in adding value to their services by improving their quality.

Although ITSM produces many benefits, organizations still face various challenges due to lack of guidelines in several contexts. This research aimed to investigate the benefits, challenges, opportunities, and implementation best practices of the ITSM domain. To conduct this research, a SLR was adopted and a discussion of results for each concept was completed. A total of 47 articles were analysed. The main contributions of this research are:

- A holistic view of ITSM implementation including how the benefits and challenges relate to each other. With this, organizations have the awareness of which benefit should be addressed first, with the purpose of achieving their main goals and avoiding undesirable challenges.

- The identification of the benefits that the organizations should focus first, to produce more benefits.

- Determination of which challenges should be avoided and considered when planning and operating the ITSM, with the view of generating as few challenges as possible to the organizations.

- The identification of main guidelines that may promote the desirable benefits in the initial phases of ITSM implementation.

- The identification of opportunities to be explored by new research, based on the difficulties that many organizations face daily.

Despite this, for future work, the challenges, benefits, opportunities, and implementation practices appointed in this research could be used to lead new investigations that would contribute not just for the ITSM domain but also for IT organizations that struggle with these issues every day. It seems that the lack of guidelines for processes improvement and assessment could be the most important "future work" in this domain, given that it is necessary for creating an improvement plan, in a view of developing mature processes that would produce the expected benefits. Considering that, research with innovative solutions for the assessment and improvement of processes should be considered. Also, a detailed examination of the relationship between benefits and challenges must be considered for new investigation. Plus, less supported concepts according to Tables $2-5$ should also be better explored in the future. For instance, which ITSM practices may exist to manage services provided through cloud technology; or how can DevOps assist ITSM discipline 
in practice (some researchers are already investigating it [24]); or provide some empirical evidences on how ITSM can help organizations reducing employees and cut costs; or even further explore and propose new maturity models to assist ITSM implementation or even reduce ITSM complexity (please see [37]).

This research has the limitation of the selected articles being from limited ranks. It is reasonable to admit that other important and relevant information could be provided by other research published in lower ranks. Additionally, this research has the limitation of the conceptual models being developed based on critical analysis, which can be criticised for several choices made.

Funding: This research received no external funding.

Institutional Review Board Statement: Not applicable.

Informed Consent Statement: Not applicable.

Data Availability Statement: Data sharing not applicable.

Conflicts of Interest: The authors declare no conflict of interest.

\section{References}

1. Melendez, K.; Dávila, A.; Pessoa, M. Information technology service management models applied to medium and small organizations: A systematic literature review. Comput. Stand. Interfaces 2016, 47, 120-127. [CrossRef]

2. Keel, A.J.; Orr, M.A.; Hernandez, R.R.; Patrocinio, E.A.; Bouchard, J. From a technology-oriented to a service-oriented approach to IT management. IBM Syst. J. 2007, 46, 549-564. [CrossRef]

3. Winniford, M.A.; Conger, S.; Erickson-Harris, L. Confusion in the ranks: IT service management practice and terminology. Inf. Syst. Manag. 2009, 26, 153-163. [CrossRef]

4. Shrestha, A.; Cater-Steel, A.; Toleman, M.; Behari, S.; Rajaeian, M.M. Development and evaluation of a software-mediated process assessment method for IT service management. Inf. Manag. 2019, 57, 103213. [CrossRef]

5. Gacenga, F.; Cater-Steel, A.; Toleman, M. An International Analysis of IT Service Management Benefits and Performance Measurement. J. Glob. Inf. Technol. Manag. 2010, 13, 28-63. [CrossRef]

6. Marrone, M.; Kolbe, L.M. Uncovering ITIL claims: IT executives' perception on benefits and Business-IT alignment. Inf. Syst. E-Bus. Manag. 2011, 9, 363-380. [CrossRef]

7. Galup, S.D.; Dattero, R.; Quan, J.J.; Conger, S. An overview of IT service management. Commun. ACM 2009, 52, 124. [CrossRef]

8. Pollard, C.; Cater-Steel, A. Justifications, Strategies, and Critical Success Factors in Successful ITIL Implementations in U.S. and Australian Companies: An Exploratory Study. Inf. Syst. Manag. 2009, 26, 164-175. [CrossRef]

9. Marrone, M.; Kolbe, L. ITIL and the Creation of Benefits: An Empirical Study on Benefits, Challenges and Processes. In Proceedings of the 18th European Conference on Information Systems, Pretoria, South Africa, 7-9 June 2010.

10. Salman, R.; Daim, T.; Raffo, D.; Dabic, M. Exploring capability maturity models and relevant practices as solutions addressing information technology service offshoring project issues. Int. J. Manag. Sci. Eng. Manag. 2018, 13, 147-157. [CrossRef]

11. Hochstein, A.; Tamm, G.; Brenner, W. Service Oriented IT Management: Benefit, Cost and Success Factors. In Proceedings of the European Conference on Information Systems, Regensburg, Germany, 26-28 May 2005; Volume 98, p. 12.

12. Koch, H.; Gierschner, C. Advantages of an ITIL-based Process Framework in a Complex SAP ${ }^{\circledR}$ System Landscape. In Proceedings of the 2007 4th IEEE Workshop on Intelligent Data Acquisition and Advanced Computing Systems: Technology and Applications, Dortmund, Germany, 6-8 September 2007; pp. 431-433.

13. Göbel, H.; Cronholm, S.; Seigerroth, U. Towards an agile method for ITSM self-assessment: A Design Science Research Approach. In Proceedings of the International Conference on Management, Leadership and Governance (ICMLG2013), Bangkok, Thailand, 7-8 February 2013.

14. Küller, P.; Vogt, M.; Hertweck, D.; Grabowski, M. A domain specific IT service management approach for small \& medium enterprises: A Domain Specific Approach. In Proceedings of the 16th International Business Information Management Association Conference, IBIMA 2011, Kuala Lumpur, Malaysia, 29-30 June 2011; Volume 3, pp. 1356-1369.

15. Jantti, M. Improving Incident Management Processes in Two IT Service Provider Companies. In Proceedings of the $201122 n d$ International Workshop on Database and Expert Systems Applications, Toulouse, France, 29 August-2 September 2011; pp. 26-30.

16. Hoerbst, A.; Hackl, W.O.; Blomer, R.; Ammenwerth, E. The status of IT service management in health care-ITIL in selected European countries. BMC Med. Inf. Decis. Mak. 2011, 11, 76. [CrossRef]

17. Machado, R.F.; Reinehr, S.; Malucelli, A. Towards a Maturity Model for IT Service Management Applied to Small and Medium Enterprises. Eur. Conf. Softw. Process Improv. 2012, 301, 157-168.

18. Gehrmann, M. Combining Itil, Cobit and Iso/Iec 27002 for Structuring Comprehensive Information Technology for Management in Organizations. Navus Rev. Gestão Tecnol. 2012, 2, 53-65. [CrossRef] 
19. Conger, S.; Winniford, M.; Erickson-Harris, L. Service Management in Operations. In Proceedings of the Americas Conference on Information Systems, Toronto, ON, Canada, 14-17 August 2008; p. 11.

20. Cater-Steel, A.; Tan, W.-G.; Toleman, M. Challenge of adopting multiple process improvement frameworks. In Proceedings of the 14th European Conference on Information Systems (ECIS 2006), Göteborg, Sweden, 12-14 June 2006; pp. $1375-1386$.

21. Pereira, R.; da Silva, M. A Literature Review: Guidelines and Contingency factors. In Proceedings of the European, Mediterranean \& Middle Eastern Conference on Information Systems, Windsor, UK, 4-5 October 2012; pp. 342-360.

22. Vicente, M.; Gama, N.; da Silva, M.M. Using archimate to represent ITIL metamodel. In Proceedings of the IEEE International Conference Business Informatics, IEEE CBI, Vienna, Austria, 15-18 July 2013; pp. 270-275.

23. Kaiser, A.K. Reinventing ITIL ${ }^{\circledR}$ in the Age of DevOps; Apress: New York, NY, USA, 2018.

24. Faustino, J.; Pereira, R.; Alturas, B.; da Silva, M.M. Agile information technology service management with DevOps: An incident management case study. Int. J. Agil. Syst. Manag. 2020, 13, 339-389. [CrossRef]

25. Sharifi, M.; Ayat, M.; Rahman, A.A.; Sahibudin, S. Lessons learned in ITIL implementation failure. In Proceedings of the International Symposium on Information Technology 2008, ITSim, Kuala Lumpur, Malaysia, 1-7 March 2008; Volume 1, pp. 8-11.

26. Beachboard, J.; Conger, S.; Galup, S.D.; Hernandez, A.; Probst, J.; Venkataraman, R. AMCIS 2007 Panel on IT Service Management: IT Service Management in the IS Curriculum. CAIS 2007, 20, 35. [CrossRef]

27. Braun, C.; Winter, R. Integration of IT Service Management into Enterprise Architecture. In Proceedings of the 2007 ACM Symposium on Applied Computing, Seoul, Korea, March 2007; pp. 1215-1219.

28. Winkler, T.J.; Wulf, J. Effectiveness of IT Service Management Capability: Value Co-Creation and Value Facilitation Mechanisms. J. Manag. Inf. Syst. 2019, 36, 639-675. [CrossRef]

29. Wan, J.; Jones, J.D. Managing IT service management implementation complexity: From the perspective of the Warfield Version of systems science. Enterp. Inf. Syst. 2013, 7, 490-522. [CrossRef]

30. Marrone, M.; Kolbe, L.M. Impact of IT Service Management Frameworks on the IT Organization: An Empirical Study on Benefits, Challenges, and Processes. Bus. Inf. Syst. Eng. 2011, 3, 5-18. [CrossRef]

31. Salle, M. IT Service Management and IT Governance: Review, Comparative Analysis and their Impact on Utility Computing; HewlettPackard Co.: Palo Alto, CA, USA, 2004; p. 26.

32. McNaughton, B.; Ray, P.; Lewis, L. Designing an evaluation framework for IT service management. Inf. Manag. 2010, 47, 219-225. [CrossRef]

33. Cots, S.; Casadesús, M.; Marimon, F. Benefits of ISO 20000 IT service management certification. Inf. Syst. E-Bus. Manag. 2016, 14, 1-18. [CrossRef]

34. Proehl, T.; Erek, K.; Limbach, F.; Zarnekow, R. Topics and Applied Theories in IT Service Management. In Proceedings of the 2013 46th Hawaii International Conference on System Sciences, Wailea, HI, USA, 7-10 January 2013; pp. 1367-1375.

35. Shrestha, A.; Cater-Steel, A.; Toleman, M.; Rout, T. Benefits and relevance of International Standards in a design science research project for process assessments. Comput. Stand. Interfaces 2018, 60, 48-56. [CrossRef]

36. Arcilla, M.; Calvo-Manzano, J.A.; Feliu, T.S. Building an IT service catalog in a small company as the main input for the IT financial management. Comput. Stand. Interfaces 2013, 36, 42-53. [CrossRef]

37. Aguiar, J.; Pereira, R.; Vasconcelos, V.B. An Overlapless Incident Management Maturity Model for Multi-Framework Assessment (ITIL, COBIT, CMMI-SVC). IJIKM 2018, 13, 137-163. [CrossRef]

38. Orta, E.; Ruiz, M. Met4ITIL: A Process Management and Simulation-based Method for Implementing ITIL. Comput. Stand. Interfaces 2018, 61, 1-19. [CrossRef]

39. Brereton, P.; Kitchenham, B.A.; Budgen, D.; Turner, M.; Khalil, M. Lessons from applying the systematic literature review process within the software engineering domain. J. Syst. Softw. 2007, 80, 571-583. [CrossRef]

40. Snyder, H. Literature review as a research methodology: An overview and guidelines. J. Bus. Res. 2019, 104, 333-339. [CrossRef]

41. Linnenluecke, M.K.; Marrone, M.; Singh, A.K. Conducting systematic literature reviews and bibliometric analyses. Aust. J. Manag. 2020, 45, 175-194. [CrossRef]

42. Okoli, C.; Schabram, K. Working Papers on Information Systems A Guide to Conducting a Systematic Literature Review of Information Systems Research. Work. Pap. Inf. Syst. 2010, 10, 10-26.

43. Mallett, R.; Hagen-Zanker, J.; Slater, R.; Duvendack, M. The benefits and challenges of using systematic reviews in international development research. J. Dev. Eff. 2012, 4, 445-455. [CrossRef]

44. Kitchenham, B. Procedures for Performing Systematic Reviews; Joint Technical Report; Computer Science Department, Keele University: Keele, UK; National ICT Australia Ltd.: Sydney, Australia, 2004.

45. Wickboldt, J.A.; Bianchin, L.A.; Lunardi, R.C.; Granville, L.Z.; Gaspary, L.P.; Bartolini, C. A framework for risk assessment based on analysis of historical information of workflow execution in IT systems. Comput. Netw. 2011, 55, 2954-2975. [CrossRef]

46. Marrone, M.; Gacenga, F.; Cater-Steel, A.; Kolbe, L. IT Service Management: A Cross-national Study of ITIL Adoption. CAIS 2014, 34, 49. [CrossRef]

47. Lucio-Nieto, T.; Colomo-Palacios, R.; Soto-Acosta, P.; Popa, S.; Amescua-Seco, A. Implementing an IT service information management framework: The case of COTEMAR. Int. J. Inf. Manag. 2012, 32, 589-594. [CrossRef]

48. Cater-Steel, D.A.; Toleman, P.M.; Tan, D.W.-G. Transforming IT Service Management-the ITIL Impact. In Proceedings of the Australasian Conference on Information Systems, Brisbane, Australia, 10-13 December 2006; p. 11. 
49. Kubiak, P.; Rass, S. An Overview of Data-Driven Techniques for IT-Service-Management. IEEE Access 2018, 6, 63664-63688. [CrossRef]

50. Tan, W.-G.; Cater-Steel, A.; Toleman, M.; Seaniger, R. Implementing Centralised IT Service Management: Drawing Lessons from the Public Sector. In Proceedings of the 18th Australasian Conference on Information Systems, Toowoomba, Australia, 5-7 December 2007.

51. Barafort, B.; di Renzo, B.; Merlan, O. Benefits Resulting from the Combined Use of ISO/IEC 15504 with the Information Technology Infrastructure Library (ITIL). In Proceedings of the International Conference on Product Focused Software Process Improvement, Oulu, Finland, 20-22 June 2002; Volume 2559, pp. 314-325.

52. Barafort, B.; Mesquida, A.-L.; Mas, A. Integrating risk management in IT settings from ISO standards and management systems perspectives. Comput. Stand. Interfaces 2017, 54, 176-185. [CrossRef]

53. Garg, S.; Misra, A. Automation of Incident Management Processes and Benefits of Hosting Servers on Cloud. In Proceedings of the International Conference on Current Trends in Computer, Electrical, Electronics and Communication, Dalian, China, 19-21 December 2017; pp. 700-702.

54. Silva, R.; Pereira, R.; Riberiro, R. Machine learning in incident categorization automation. In Proceedings of the Iberian Conference on Information Systems and Technologies, Caceres, Spain, 13-16 June 2018; pp. 1-6.

55. Heikkinen, S.; Jäntti, M. Identifying IT Service Management Challenges: A Case Study in Two IT Service Provider Companies. In Proceedings of the 2012 23rd International Workshop on Database and Expert Systems Applications, Vienna, Austria, 3-6 September 2012; pp. 55-59.

56. McBride, N. Exploring service issues within the IT organisation: Four mini-case studies. Int. J. Inf. Manag. 2009, 29, 237-243. [CrossRef]

57. Asrowardi, I.; Putra, S.D.; Subyantoro, E.; Daud, N.H.M. IT Service Management System Measurement using ISO20000-1 and ISO15504-8: Developing a Solution-Mediated Process Assessment Tool to \{Enable Transparent and SMS Process Assessment. IJECE 2018, 8, 4023. [CrossRef]

58. Shrestha, A.; Cater-Steel, A.; Toleman, M.; Tan, W.-G. Building a Software Tool for Transparent and Efficient Process Assessments in IT Service Management. Int. Conf. Des. Sci. Res. Inf. Syst. 2014, 8463, 241-256.

59. Tan, W.-G.; Cater-Steel, A.; Toleman, M. Implementing IT service management: A case study focussing on critical success factors. J. Comput. Inf. Syst. 2010, 50,1-12.

60. Shrestha, A.; Cater-Steel, A.; Toleman, M.; Tan, W.-G. A Decision Support Tool to Define Scope in IT Service Management Process Assessment and Improvement. Int. Conf. Des. Sci. Res. Inf. Syst. 2013, 7939, 308-323.

61. Deutscher, J.; Felden, C. Model Concept to Determine the Optimal Maturity of IT Service Management Processes. In Proceedings of the 2009 Eighth IEEE/ACIS International Conference on Computer and Information Science, Shanghai, China, 1-3 June 2009; pp. 543-548.

62. Jia, R.; Reich, B.H. IT service climate, antecedents and IT service quality outcomes: Some initial evidence. J. Strateg. Inf. Syst. 2013, 22, 51-69. [CrossRef]

63. Pereira, R.; da Silva, M.M. A maturity model for implementing ITIL V3 in practice. In Proceedings of the IEEE International Enterprise Distributed Object Computing Workshop, EDOC, Helsinki, Finland, 29 August-2 September 2011; pp. 259-268.

64. De Sousa Pereira, R.F.; da Silva, M.M. A maturity model for implementing ITIL v3. In Proceedings of the 2010 6th World Congress on Services, Miami, FL, USA, 5-10 July 2010; pp. 399-406.

65. Richter, C.; Schaaf, T. A maturity model for tool landscapes of IT service providers. In Proceedings of the 12th IFIP/IEEE International Symposium on Integrated Network Management, Dublin, Ireland, 23-27 May 2011; pp. 1050-1057. 